\title{
A Child with Ulcerative Colitis - A Case Report
}

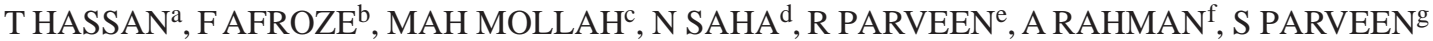

\begin{abstract}
Summa1ry:
Ulcerative colitis in children has not been reported previously in Bangladesh. This case report is of a 7 year old girl who presented with frequent passage of slimy stool mixed with blood for 4 months. She also had fever, weight loss, abdominal pain and urgency at defecation. Barium enema showed pancolitis with multiple pseudopolyps and collar button appearance. Colonoscopy revealed a friable and granular mucosa with diffuse ulceration and multiple pseudopolyps. There was bloody
\end{abstract}

\section{Introduction:}

Ulcerative colitis (UC), a chronic inflammatory bowel disease of the colon is not uncommon in children ${ }^{1}$. The incidence of UC is 2 to 11 per 100,000 children per year ${ }^{2}$. Median age at diagnosis is 10 years ${ }^{3}$. Multiple familial occurrences are present in $15-20 \%$ of patients with no sex predilection ${ }^{4}$. It is thought to be a multifactorial disease. Both genetic and environmental influences are present in the pathogenesis of the disease ${ }^{5}$. Though it is seen frequently in Europe and North America but studies have shown that the incidence is more or less same in developed and developing countries ${ }^{6}$. The increased incidence is related to the rapid

a. Dr. Tariq Hassan, FCPS (Paed), Diploma in Paediatric Gastroenterology (UK), Assistant Professor, Department of Paediatrics, Dhaka Medical College and Hospital

b. Dr. Farzana Afroze, M.B.B.S., Honarary Medical Officer, Department of Paediatrics, DMCH

c. Dr. Md. Abid Hossain Mollah, FCPS (Paed), Diploma in Medicine (UK), FACP (Usa), FRCP (Edin. UK), Professor And Head, Department of Paediatrics, DMCH

d. Dr. Narayan Saha, FCPS (Paed), Associate Professor, Department of Paediatrics, DMCH

e. Dr. Rubaiya Parveen, FCPS (Paed), Registrar, Department of Paediatrics, DMCH

f. Dr. Anisur Rahman, M.B.B.S., Assistant Registrar, Department of Paediatrics, DMCH

g. Dr. Shahana Parveen, M.B.B.S., Assistant Registrar, Department of Paediatrics, DMCH

Address of Correspondence: Dr. Tariq Hassan, FCPS (Paed), Diploma in Paediatric Gastroenterology (Uk), Assistant Professor, Department of Paediatrics, DMCH, E-mail: tariq-dmc @ yahoo.com Received: 20 April, 2008 Accepted: 10 February, 2009 exudate and the lumen was narrow. Histopathology findings showed necrosed tissue probably representing a base of ulcer and inflammatory cell infiltrate in the lamina propria. The crypt architecture was distorted with cryptitis and goblet cell depletion. There was no granuloma. She was diagnosed as a case of ulcerative colitis \& treated with Sulfasalazine and oral corticosteroid. Her response to treatment was very well and now she is on regular follow up.

(J Bangladesh Coll Phys Surg 2010; 28: 117-120)

westernization of lifestyles as well as environmental changes caused by industrialization and urbanization ${ }^{6,7}$. There are no reported cases or data from Bangladesh on this particular problem. The diagnosis, management, course and prognosis of the disease remain highly enigmatic. In view of the situation, the case is reported here for rarity.

\section{Case summary:}

A 7 year old girl was admitted in pediatric department of Dhaka Medical College Hospital on 22/07/07 with frequent passage of slimy stool mixed with blood for the last four months. The frequency was about 8-10 times per day. Additional symptoms included anorexia, tenesmus, and abdominal pain, urgency at defecation, fever and significant weight loss. For this she was seen by local physicians and treated with antibiotics on several occasions but her condition did not improve. She had no food allergies, alteration of bowel habits. She had no contact with tuberculosis neither her family history was notable.

On physical examination she looked emaciated, pale, and was febrile $\left(101^{\circ} \mathrm{F}\right)$. Her heart rate was 100 beats / $\mathrm{min}$, blood pressure $(90 / 60 \mathrm{~mm} \mathrm{Hg}$ ) and respiratory rate (24/min) were normal. There was no clubbing, no lymphadenopathy and no skin lesion. Her height was $110 \mathrm{~cm}$ ( $90 \%$ of reference value of CDC, just below $3^{\text {rd }}$ centile), and weight was $13 \mathrm{~kg}$ ( $56.5 \%$ of reference value of $C D C$, far below $3^{\text {rd }}$ centile). Her oral mucosa was dry. Abdomen was slightly distended and diffusely tender but there was no rebound tenderness or guarding. No abnormality was noted in the perianal region as well 
as in the joints. Other systemic examination revealed normal.

Initial investigations showed Hb- 50\%, white cell count9000/cu mm with normal differential and platelet count. Peripheral blood film showed microcytic hypochromic anemia, E.S.R was $80 \mathrm{~mm}$ in $1^{\text {st }}$ hour. Her serum albumin level $(2.4 \mathrm{gm} / \mathrm{dl})$ was low. Stool microscopy showed plenty of red cells and leukocytes. Stool culture was negative. Her chest X-ray was normal. Abdominal ultrasound revealed normal study. Barium enema showed pancolitis with multiple pseudopolyps and collar button appearance.

A colonoscopy was done up to the hepatic flexure which

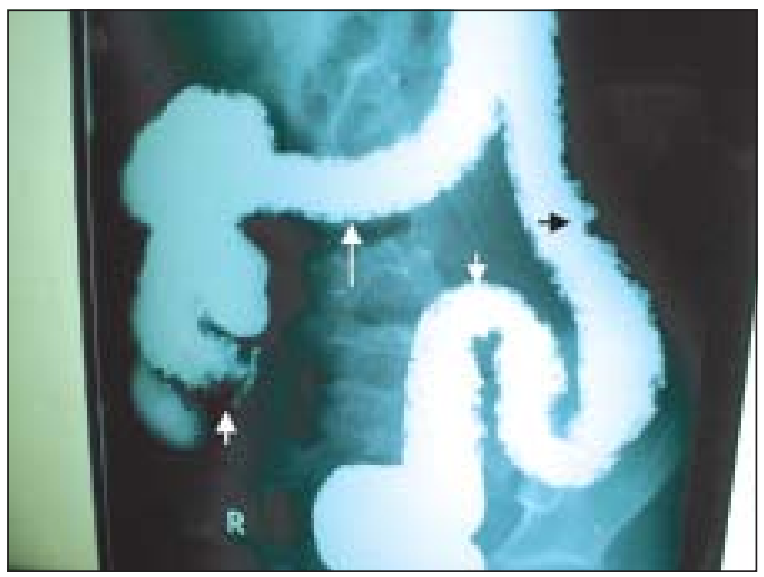

Fig. 1: Barium enema showing serrated thorn like colonic outlines indicating microulceration which diffusely involved the whole colon. Pseudopolyps and collar button appearance signifying submucosal tracking also seen.

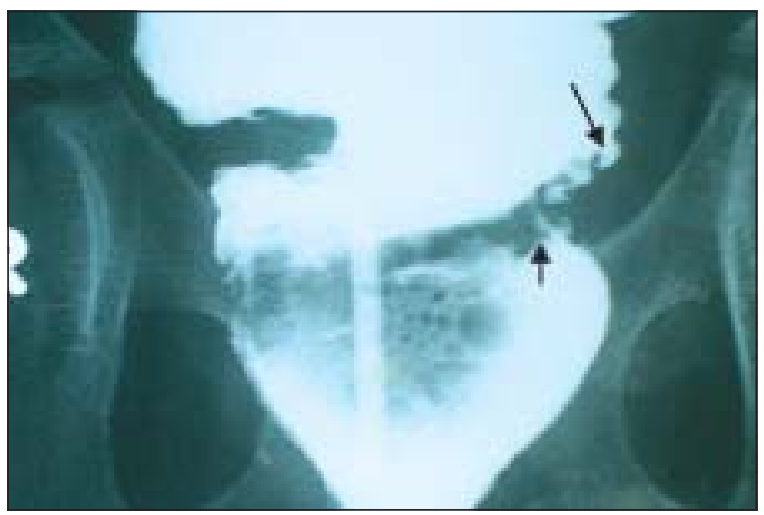

Fig.2: Barium enema showing multiple pseudopoly, and collar button appearance (upper arrow). showed diffuse ulceration with multiple Pseudopolyps involving extensively from rectum to hepatic flexure. The colonic mucosa was erythematous friable and granular. There was bloody exudate and the lumen was narrow. The findings were consistent with severe colitis. Biopsy specimens showed necrosed tissue probably representing a base of ulcer and inflammatory cell infiltrate in the lamina propria. The crypt architecture was distorted with cryptitis and goblet cell depletion. There was no granuloma. So the diagnosis of Ulcerative colitis was made. She was treated with steroid and sulfasalazine from the $6^{\text {th }}$ day of admission. Her symptoms of colitis begun to improve from the $3^{\text {rd }}$ week of treatment and subsequently she was discharged home on steroid and sulfasalazine. Her bowel movement had normalized by the time of her out patient review 4 weeks later. The girl is now on regular follow up.

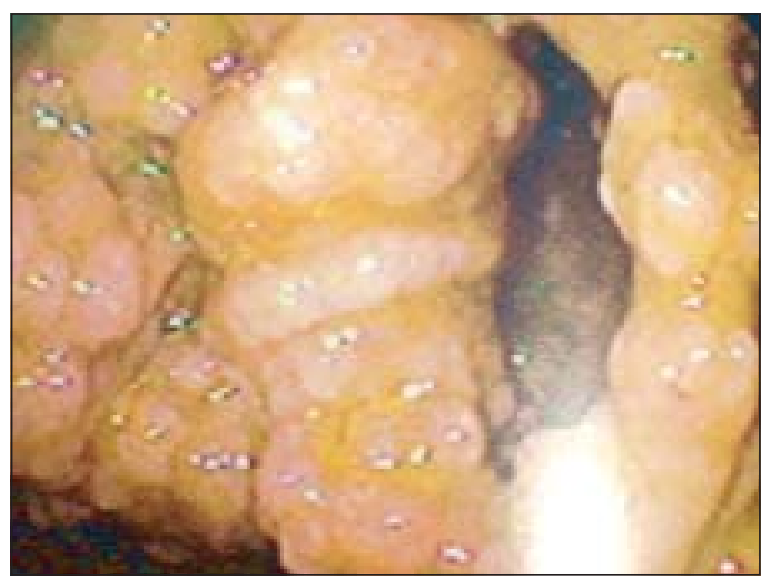

Fig.-3: endoscopic image demonstrating multiple Pseudopolyps.

\section{Discussion:}

Ulcerative colitis (UC) is a chronic inflammatory bowel disease of the colon characterized by remission and relapse $^{5}$. UC and Crohn's disease comprise the spectrum of inflammatory bowel disease. It is thought to be a multifactorial disease. Both genetic and environmental influences are present in the pathogenesis of the disease ${ }^{5}$. An abnormality in intestinal mucosal immunoregulation may play an important role. Normally gut mucosa displays physiologic inflammation in response to constant immunologic stimulation from microbial agents and dietary antigens. In UC the mechanism that keeps 
physiological inflammation in check fail and pathologic inflammation ensues. Inflammatory mediators like cytokines, arachidonic acid metabolites, reactive oxygen metabolites, growth factors etc are involved, leading to tissue destruction and remodeling with fibrosis. Most therapies are aimed at interfering with these mediators 5 .

The hall mark symptoms are chronicity (>2-3 wks), abdominal cramping, diarrhea and bloody stool ${ }^{5,8}$. In $50-60 \%$ cases the disease is mild affecting the distal colon only with no systemic manifestation ${ }^{8}$. Moderate disease is observed in $30 \%$ of patients with bloody diarrhea, cramps, urgency to defecate and abdominal tenderness. Associated systemic findings such as anorexia, weight loss, low grade fever and mild anemia are present ${ }^{8,11,13}$. About $10 \%$ patients present with features of severe colitis such as more then six bloody stools per day, fever, weight loss, abdominal tenderness, anemia, leucocytosis, hypoalbuminaemia. Life threatening complications like severe hemorrhage, toxic megacolon, or intestinal perforation may occur in these patients. Similarly this patient had slimy stool mixed with blood more than six times a day for four months, fever, weight loss, abdominal tenderness, anemia and hypoalbuminemia. But she did not have any complication. Very few $(<5 \%)$ cases present with predominantly extra intestinal manifestations like growth failure, arthropathy, skin manifestations (pyoderma gangrenosum) or liver disease (Sclerosing cholangitis) ${ }^{8}$.

Diagnosis of UC is based on history, clinical examination and typical endoscopic and histologic findings ${ }^{5}$. Infectious colitis can mimic UC, so all patients should have stool culture and stool evaluation for ova and parasites ${ }^{5}$. Distinguishing UC and Crohns disease (CD) is essential because treatments and anticipated complications will differ. Blood in stool is usually but not always characteristic of UC whereas CD usually present as non bloody diarrhea, extraintestinal manifestations such as clubbing, oral ulceration, fever, arthritis, erythema nodosum, growth retardation etc and perianal disease including skin tags, anal fissures, and fistulas are more common in $\mathrm{CD}^{3,5,9}$. Moreover endoscopic examination shows that UC often begins in the rectum and spread proximally and continuously, Pseudopolyps occur commonly, where as CD often skip some areas of bowel and spare the rectum as many as $50 \%$ of cases $^{2,3}$. Beside this the severity of UC can be judged by colonoscopy. In mild cases only a small area of distal colon is involved. In severe cases there can be pan colitis. Although in this patient colonoscopy was performed up to hepatic flexure, usually a full colonoscopy is indicated to examine the entire colon for discontinuous areas of inflammation (Known as skip lesion) and to visualize the terminal colon. In this girl the barium enema revealed pancolitis and the colonoscopy showed diffuse ulceration with multiple Pseudopolyps involving extensively from rectum to hepatic flexure. There was no skip lesion.In UC biopsy specimen shows distortion of crypts, inflammatory cell infiltrate in lamina propria, cryptitis, crypt abscesses and goblet cell depletion. In CD the involvement is transmural and non caseating granuloma is characteristic ${ }^{9}$. This patient's biopsy specimens showed necrosed tissue probably representing a base of ulcer and inflammatory cell infiltrate in the lamina propria, there was no granuloma. Additionally it is often difficult to distinguish between UC and CD both endoscopically and histologically, which then diagnosed as indeterminate colitis. About $10 \%$ of affected patients will have an indeterminate colitis ${ }^{5}$. Allergic colitis also mimics UC, but usually presents in infancy and resolve on removal of offending protein.

The therapy for UC occurs in two steps. The first step is to induce remission and second step is to maintain remission ${ }^{3,11}$. The mainstay of outpatient management of patient with mild to moderate colitis is antiinflammatory therapy with Aminosalicylates like Sulfasalazine, Mesalamine ${ }^{5,8}$. The dose of Sulfasalazine is $50-75 \mathrm{mg} / \mathrm{kg} /$ day (max.2 to $3 \mathrm{~g} /$ day). Onset of action may take several weeks ${ }^{5}$. The patients who have proctitis or left sided colitis only, oral and topical formulations are equally effective in achieving symptomatic control and inducing remission ${ }^{3,8,12}$. But patients with mild to moderate pancolitis topical therapy are not an effective sole treatment modality because enemas rarely pass the splenic flexure ${ }^{2}$. The exact mechanism of action of Aminosalicylates in ulcerative colitis is unclear. However it causes modulation of prostaglandin pathways and also inhibits the production of potent inflammatory cytokines such as IL-1, TNF and interferon $-\tilde{a}^{5}$. Hypersensitivity is the major side effect, other dose related adverse reactions include nausea, vomiting, headache etc. ${ }^{2,5}$. Mesalamine may have fewer 
side effects but it is not available in a pediatric preparation ${ }^{8}$. In case of severe colitis like our patient oral or IV corticosteroid must be used to induce remission and continue until symptoms abate and bowel movement normalize. The dose is then tapered gradually to an alternate day dose within 1-3 month. With medical management most child are in remission within three months ${ }^{5}$. The level of therapy that induced remission dictates the selection of maintenance therapy. If steroid is used to induce remission large doses of Aminosalicylates may be required to prevent relapse as steroid tapered $d^{3,8}$. Priya was treated with Sulfasalazine and oral steroid. With this treatment she responded well. At a follow up visit 6 weeks later she reported that her diarrhea had completely resolved. There was no visible blood in the stool. She had no fever or abdominal pain. Her lab parameters also became normal. But endoscopy was not done to see the remission. Now she is on regular follow up.

Other modalities of therapy for steroid dependent or steroid resistant cases include 6-MC, Azathioprin, cyclosporine, infliximab. Surgery (colectomy) is curative in UC and performed for intractable disease, complication of therapy and fulmina ${ }^{2,3,14}$. Regular follow up is essential in these patients as the principal long term complications are primary sclerosing cholangitis and colon cancer. The annual rate of development of colon cancer is $2 \%$ after 20 years of disease and $8 \%$ after 30 years $^{3}$. The risk can be diminished with surveillance colonscopy begaining 810 years of disease and detaction of significant dysplasia on biopsy would prompt colectomy.

\section{References:}

1. Devlin HB, Datta D, Dellipiani AW. The incidence and prevalence of inflammatory bowel disease in North Tees Health District. World J Surg 1990; 4: 183-93

2. Robert R, Jhon $\mathrm{H}$ et al. Medical and Surgical Management of Chronic Ulcerative Colitis. Arch SURG. 2005 Mar; 140:380391.
3. Brayn AL, Paul TK, Slephen BH. Ulcerative Colitis: Diagnosis and Management. Hospit Physicia 2002 Jun; 38(6):53-63.

4. Selby W: The natural history of ulcerative colitis. Baillieres Clin Gastroenterol 1997 Mar; 11(1): 53-64.

5. Behrman RE, Kligman RM, Jonson HB. Nelson Text Book of Pediatrics. 17 $7^{\text {th }}$ ed. Philadelphia: W B Saunders; 2004.

6. Soda A, Midha V , Sood N, Bhatia AS et al. Incidence and prevalence of ulcerative colitis in Punjab, north India. Gut 2003; 52:1587-1590.

7. Lee YM, Fock km et al. Racial differences in the prevalence of Ulcerative colitis and Crohn's disease in Singapore. J Gastroenterol Hepatol. 2000 Jun; 15(6):622-625.

8. Jonathon MW, Peter M, Robert B, David P. Ulcerative colitis. [Online] 2007 Mar. [cited 2007 Aug]. Available from: URL: http://www.eMedicine.com.

9. Sanders DS: The differential diagnosis of Crohn's disease and ulcerative colitis. Baillieres Clin Gastroenterol 1998 Mar; 12(1): 19-33.

10. Rajwal SR, Puntis JW, McClean P, et al: Endoscopic rectal sparing in children with untreated ulcerative colitis. J Pediatr Gastroenterol Nutr 2004 Jan; 38(1): 66-69.

11. Kirschner BS .Ulcerative colitis in children.Pediatr Clin North Am 1996; 43:235-54.

12. Kamm MA, Senapati A. Drug management of ulcerative colitis.BMJ 1992; 305:35-9.

13. Leichtner AM, Jackson WD, Grand RJ.Ulcerative colitis.IN: Walker WA, Durie PR, Hamilton JR, etal, editors. Pediarric gastrointestinal disease: pathophysiology, diagnosis, management.2nd ed. St Louis: Mosby; 1996:712-23.

14. Ward FM, Bodger K, Daly MJ, Heatley RV. Clinical economics review: medical management of inflammatory bowel disease. Aliment Pharmacol Ther 1999:13; 15-25.

15. Stein RB, Hanauer SB.Medical therapy for inflammatory bowel diseAm 1999; 28:297-321.

16. Sachar DB.Maintenance therapy in ulcerative colitis and Crohn’s disease.J Clin Gastroenterol 1995; 20:117-22.

17. Hyde GM, Jewell DP. Review article: the management of severe ulcerative colitis. Aliment Pharmacol ther 1997; 11: 419-24. 